\title{
Pflege im Dialog
}

\section{Praxisfragen und Forschungskonzepte}

Die Dialogreihen „Pflegeforschung und Praxis“ und „PflegepraxisPolitik-Management-Lehre" bieten als Kooperation zwischen dem Institut für Pflegewissenschaft der Universität Wien und der Akademie für Fortbildungen und Sonderausbildungen Bereich Pflege des AKH Wien eine kostenlose Informationsvermittlung mit Möglichkeit zur Diskussion.

Im ersten Halbjahr stehen im Jugendstilhörsaal der MedUni Wien noch drei Veranstaltungen auf dem Programm, die die Gesundheits- und Krankenpflege nicht zuletzt in der Vernetzung der Aufgaben darstellen.

\section{Geburtshilfe: Stille Geburt}

Die Situation von Pflegepersonen einer gynäkologischen Normalstation in der spezifischen Betreuungssituation rund um einen Spätabort bzw. eine Totgeburt stellt Lisa Brunhuber, BSc, MA, DGKS im SMZ Süd - KFJ, dar. Sie präsentiert die Ergebnisse einer Studie, die zeigt, dass sich die Pflegepersonen im Dilemma zwischen dem Anspruch „Als Pflegeperson das Wohl der Patientin in den Mittelpunkt stellen" und der persönlich belastenden Situation befinden. Termin: 27.4.2016, 16:30 bis 18:00 Uhr

\section{Praxisfragen als Modell}

Die Fragen, die sich in der Praxis ergeben, sind vielfältig. Maria Stockmayr, BScN, Pflegeberaterin an der Universitätsklinik für Chirurgie, AKH-Wien, zeigt anhand konkreter Fälle in ihrem täglichen Arbeitsbereich das Modell „Praxisfragen“, dessen Wahrnehmung in der Praxis und die mögliche Funktion als Brücke zwischen Theorie und Praxis.

Termin: 18.5.2016, 16:30 bis 18:00 Uhr

\section{Symptomtagebuch für Patientinnenbetreuung}

Die Inzidenz von vulvären Neoplasien ist weltweit steigend, insbesondere auch zunehmend bei jüngeren Frauen. Zur Vermeidung von fortschreitenden invasiven Erkrankungen ist der chirurgische Eingriff die Standardtherapie. Bereits bei minimal invasiven Eingriffen treten postoperativ neben körperlichen Symptomen und Nebenwirkungen insbesondere auch psychosoziale Symptome auf. Prof. Dr. Beate Senn, RN, PhD, Leiterin des Instituts für Angewandte Pflegewissenschaft, St. Gallen, und Mag. Silvia Raphaelis, Institut für Pflegewissenschaft, Universität Wien, berichten über „Das Symptomtagebuch als Beispiel eines strukturellen Instrumentes und eine Leitlinie für die Patientinnenberatung“.

Termin: 18.5.2016, 16:30 bis 18:00 Uhr

Informationen für alle Veranstaltungen: www.wienkav.at/kav/ausbildung/fba/pflege

Anmeldung für alle Termine: post_akh_sfzp@akhwien.at

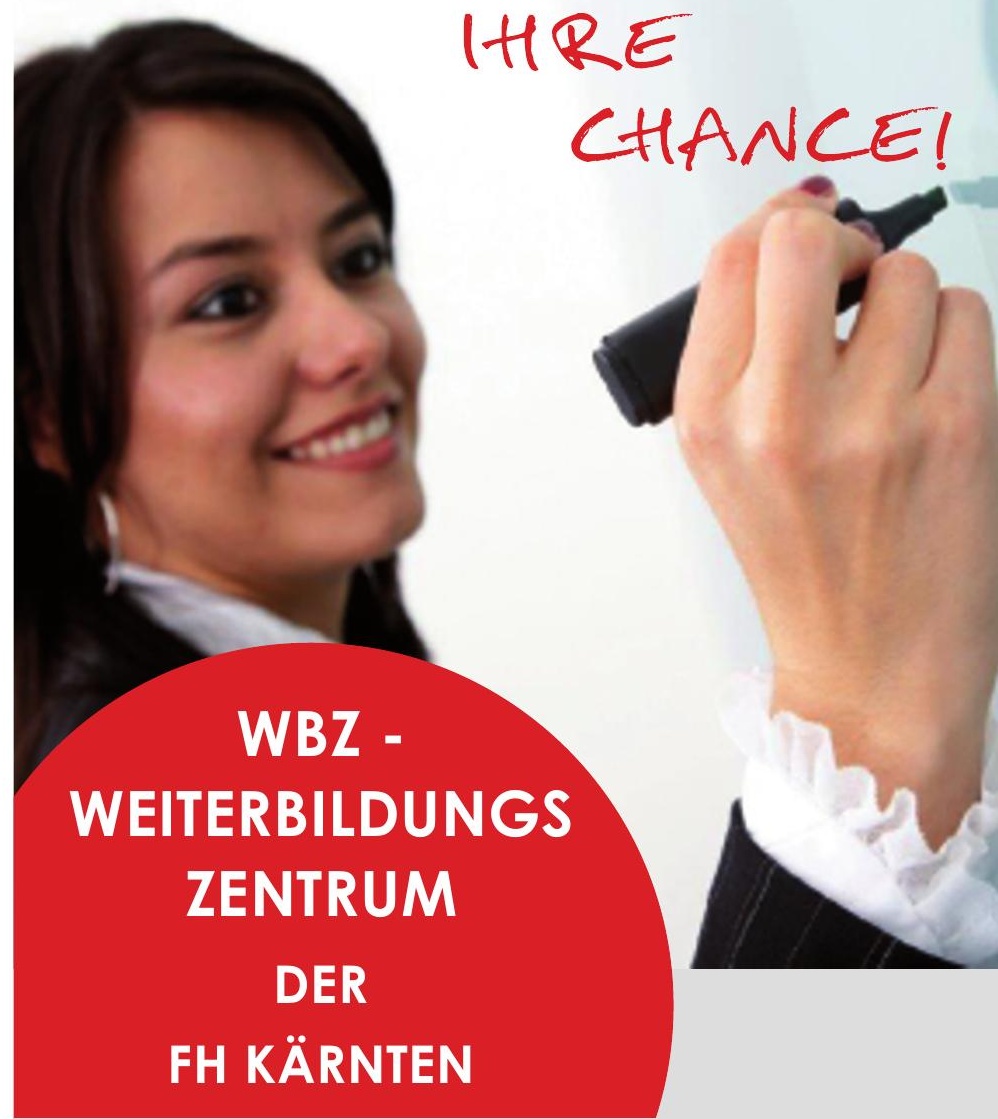

Wir laden zu unseren INFO-VERANSTALTUNGEN ein

\section{AKADEMISCHES CASE MANAGEMENT}

3 Semester, 60 ECTS, geplanter Start: Oktober 2016

Info -Veranstaltungen:

- Mittwoch, 20. April 2016 um 17:00 Uhr

- Mittwoch, 8. Juni 2016 um 16:30 Uhr

\section{AKADEMISCHES GESUNDHEITS- UND PFLEGEMANAGEMENT}

(Berufsberechtigung nach § 72GuKG)

4 Semester, 60 ECTS, geplanter Start: September 2016

Info -Veranstaltungen:

- Donnerstag, 14. April 2016 um 16:00 Uhr

- Freitag, 10. Juni 2016 um 16:30 Uhr

\section{MASTER PÄDAGOGIK FÜR GESUNDHEITSBERUFE}

(Berufsberechtigung nach § 71GuKG)

4 Semester, 120 ECTS, geplanter Start: September 2016

Info -Veranstaltungen:

- Donnerstag, 31. März 2016 um 17:00 Uhr

- Donnerstag, 28. April 2016 um 17:00 Uhr

Veranstaltungsort

Fachhochschule Kärnten WBZ - Weiterbildungszentrum,

Hauptplatz 12

9560 Feldkirchen

T.: +43 (0) 5/90500 - 4301
Aus organisatorischen Gründen bitten wir um Anmeldung per Mail mit Angabe des Lehrgangs: weiterbildung@fh-kaernten.at 